\title{
The Impact of COVID-19 Pandemic on Maternal and Child Health Services in Rwanda
}

\author{
Tengera Olive $^{1,2}$, Ndayisenga Jean Pierre ${ }^{1,2,6}$, Mukamana Donatilla ${ }^{1}$, Muhayimana Alice ${ }^{1,2}$, \\ Murekezi Josephine, ${ }^{2,3}$, Mukamusangwa Mediatrice ${ }^{2,4}$, Uwambajimana Rosalie ${ }^{2,3}$, \\ Musabe Joyce ${ }^{5}$
}

Dear Editor,

Since March 14th, 2020 the first case of the COVID19 was identified in Rwanda. Like other countries, preventive measures were introduced by the government of Rwanda (GoR) in collaboration with the Ministry of Health $(\mathrm{MoH})$, the Ministry of Local Government, the Ministry of Internal Security, the Ministry of Defense, and all stakeholders. Interestingly, all policy and decision-makers were involved in the implementation of COVID-19 preventive measures including hand hygiene, stay home, physical and social distancing. Only essential services were opened. Both private and public transportation were closed except those which were at the frontline- related services (1).

Also, borders were closed and international travels were suspended except for emergency flights and cargo. To control the rapid spreading of the COVID-19 in Rwanda,-people found with signs and symptoms of the disease, were sensitized and were told to call the emergency hotline 114 to receive treatment and quarantine in the appropriate health centers prepared to receive cases of COVID 19. In line with World Health Organization (WHO) recommendations, people who come from abroad or from one province to another, or had been in contact with those infected, were supposed to be in quarantine for 14 days (2).

The GoR through the MoH facilitated health care providers to continue serving the population within their provincial, district hospitals, and health centers. However, access to those essential services was a big challenge towards the Rwandan population. Therefore, it is imperative to say that the COVID-19 affected directly and indirectly the maternal and child health care services. Hence, women seeking maternity care-related services were faced with significant challenges by limited transport options to health care services. Only private cars were allowed to be used under permission of Rwanda National Police. During the lockdown period, due to the transportation challenges, many women were not able to respect their antenatal care visit scheduled appointments given to them.

Furthermore, health care providers especially midwives who provide maternity care services faced

\author{
*Corresponding Author \\ Tengera $\mathrm{O}$. \\ http://orcid.org/0000-0003-1149-2568 \\ E-mail: tengera.olive@gmail.com
}

'School of Nursing and Midwifery at University of Rwanda, Kigali, Rwanda,

${ }^{2}$ Rwanda Association of Midwives, Kigali, Rwanda, ${ }^{3}$ King Faisal Hospital, Kigali, Rwanda,

${ }^{4}$ Muhima District Hospital, Kigali, Rwanda

${ }^{5}$ Training Support Access Model project for Maternal Newborn Child Health Project-Rwanda

${ }^{6}$ Arthur Labatt Family School of Nursing, FHS, UWO 
many challenges as consequences of no adequate birth preparedness among pregnant women and their families. The issue of transport was a big challenge for healthcare providers including midwives when going to and coming from their workplaces. For instance, some midwives walked around 10 kilometers to reach the bus station.

Health care providers living in the countryside were obliged to stay home due to limited access to transport. Besides, midwives were deployed in new created COVID-19 centers, causing a shortage of skilled staff and an increase in the midwives' workload.

During the lockdown, midwives and nurses were afraid due to COVID-19 not trusting that every patient who came for services was free from COVID19. Patients also were worried about seeking health services, fearing that they will be contaminated by health care providers because they are more exposed to COVID-19 from clients they serve. This affected negatively the provision of labour support lead to bad experiences in delivering mothers.

The emergency training was done to all staff on the use of PPEs. The relationship between midwife and client was interrupted due to PPEs, the trust was reduced at the level of requesting midwives to remove PPEs to know who is caring for them

Midwives are mentally affected and overwhelmed in a busy and high-risk environment that may lead to the burnout due to shortage of staff and COVID -19 exposition. When a client was suspected of being infected by COVID-19, was isolated, Therefore, the midwife who assigned to care isolated mothers were at high risks of developing anxiety of exposing their life and their family members to the COVID-19. However, midwives are coping due to measures taken by hospitals such as training on self-protection.

During COVID-19 period the triage seems to be delayed due to the temperature checking and history taking for all clients. The suspected would be isolated and the literature says that this may lead to the clients feeling stigmatized.

In general, the COVID-19 restrictive preventive measures have negatively affected maternal and child health care services. It is recommended that the policymakers and decisions makers could consider the role of midwives in the provision of respectful maternity care during the COVID-19 pandemic period. Hence, special consideration is needed such as the provision of transport means to all healthcare providers including midwives. Also, respectful maternity care could be maintained and considered by all stakeholders to promote the best maternity outcomes.

\section{ACKNOWLEDGEMENT}

We acknowledged the contribution of the above authors on this letter to the Editor.

Funding: None declared

Ethical Clearance: None declared

Conflict of Interest: None declared

\section{Declaration of Interests}

No competing financial interests are declared.

AM was supported by the Consortium for Advanced Research Training in Africa (CARTA). CARTA is jointly led by the African Population and Health Research Center and the University of the Witwatersrand and funded by the Carnegie Corporation of New York (Grant No-B 8606.RO2), Sida (Grant No 54100029), the DELTAS Africa Initiative (Grant No: 107768/z/15/z). The DELTAS Africa initiative is an independent funding scheme of the African Academy of Sciences (AAS)'s Alliance for Accelerating Excellence in Science in Africa (AESA) and supported by the new partnership for Africa's Development Planning and Coordinating Agency (NEPAD Agency) with funding from the Welcome Trust (UK) and the UK government. The statements made and views expressed are solely the responsibility of the fellow.

\section{REFERENCES}

1. WHO (2020) First Case of COVID-19 confirmed in Rwanda. Web page

2. WHO (2020) Coronavirus disease (COVID-19) advice for the public. Web page 